$+{ }^{+}+{ }^{+}+$La revue pour l'histoire du CNRS

POUR LHISTOIRE DU CNRS $\quad 24 \mid 2009$

Soixante-dixième anniversaire du CNRS

« Le soleil donne »

Pierre Matarasso

(2) OpenEdition

Journals

Édition électronique

URL : https://journals.openedition.org/histoire-cnrs/9047

DOI : 10.4000/histoire-cnrs.9047

ISSN : 1955-2408

Éditeur

CNRS Éditions

Édition imprimée

Date de publication : 5 octobre 2009

ISSN : 1298-9800

Référence électronique

Pierre Matarasso, « "Le soleil donne » », La revue pour l'histoire du CNRS [En ligne], 24 | 2009, mis en ligne le 05 octobre 2009, consulté le 20 mai 2021. URL : http://journals.openedition.org/histoire-cnrs/ 9047 ; DOI : https://doi.org/10.4000/histoire-cnrs.9047

Ce document a été généré automatiquement le 20 mai 2021.

Comité pour l'histoire du CNRS 


\title{
« Le soleil donne »
}

\author{
Pierre Matarasso
}

1 Lorsqu'en 1974, Robert Chabbal, alors directeur scientifique de la physique au CNRS, décide de lancer le Programme interdisciplinaire sur l'énergie solaire (Pirdes), certains événements survenus depuis la fin des années 1960 légitiment cette décision.

2 Notre vision du monde a changé depuis les premiers vols spatiaux, en particulier depuis les premiers vols habités et l'alunissage d'Apollo 11. La photographie de la Terre, flottant dans le vide intersidéral, a émerveillé les esprits mais aussi éveillé les consciences: nous vivons sur une planète finie dont le fonctionnement global est affecté par les activités humaines.

3 L'architecte Buckminster Fuller et l'économiste Kenneth Boulding ont exprimé à la fin des années 1960 cette conception dans des écrits sur le Spaceship Earth. L'idée qu'ils expriment d'un Système Terre qui peut être comparé à un vaisseau spatial, s'appuie à la fois sur la vision de notre planète depuis l'espace, les progrès de la géochimie et l'essor des idées systémiques. Nous vivons sur des stocks d'énergies et de matériaux fossiles; l'avenir nous imposera tôt ou tard de nous intégrer dans les «flux » dont le plus exemplaire, est celui de l'énergie solaire qui baigne la Terre.

4 L'énergie solaire avait déjà, depuis les années 1950, fait l'objet d'un développement au CNRS. Avec comme pionnier, Félix Trombe, à l'origine du grand four solaire installé par le CNRS à Odeillo dans les Pyrénées. Le grand four solaire était à l'origine davantage destiné à la chimie des hautes températures qu'à la production d'énergie. Ce grand instrument, contemporain des premiers accélérateurs linéaires, montre que physique fondamentale et énergies naturelles ne sont pas antinomiques. Le CNRS dispose donc au début des années 1970 d'une expertise optique, thermique et d'engineering sur le solaire grâce à Félix Trombe et d'autres chercheurs comme Georges Perri à Marseille ou Michel Rodot qui, dans son laboratoire du site de Meudon Bellevue, poursuit des recherches sur les photopiles (cellules photovoltaïques) porteuses de beaucoup d'espoir (en particulier grâce à leurs utilisations spatiales). Du côté de l'Anvar, Jean Deflandre ${ }^{1}$, délégué scientifique, avait commencé dans les années 1970 à soutenir un jeune industriel humaniste qui avait mis au point, dans l'entreprise familiale Girardier, une pompe solaire thermodynamique, destinée aux zones isolées du Sahel dont l'un des 
prototypes fut installé dans un village mauritanien. Pour ma part, j'avais réalisé pour un périodique du groupe de l'Usine nouvelle, le journal Phase Zéro, une prospective sur l'énergie solaire dans le numéro spécial Innovation 1972. Je m'intéressais en particulier à deux applications futuristes: les grandes centrales solaires thermodynamiques à concentration et les centrales en orbites terrestres proposées par le cabinet Arthur. D. Little, spécialisé dans l'innovation. À l'occasion de mon entrée à l'Anvar, j'y rencontrais Jean Deflandre et il nous parut vite évident que les recherches sur l'énergie solaire n'étaient pas à la hauteur des enjeux énergétiques futurs.

Les partisans de l'énergie solaire voyaient là le prolongement d'une exploitation des énergies naturelles de "flux » plutôt que celle des énergies de «stocks » (combustibles fossiles ou nucléaires). Cette conception était dans le prolongement direct d'une tradition qui avait été celle de la « houille blanche » et de l'époque des grands barrages producteurs d'électricité par EDF. Pour un certain nombre d'acteurs de l'époque (dont Jean Deflandre et moi-même), cette question possédait une légitimation géophysique, liée à la conscience de l'épuisement des ressources et aux émissions de $\mathrm{CO}_{2}$. Rappelons l'émergence politique de la question du changement climatique dès 1970, la vérification aux États-Unis de la théorie du Peak oil, le premier rapport au Club de Rome Limits to Growth qui sont à peu près contemporains de "la première crise de l'énergie ». C'est dans ce contexte que Jean Deflandre prend contact avec Jean Lagasse, directeur du Laas (l'un des grands laboratoires du CNRS) et fervent promoteur des sciences de l'ingénieur. Celui-ci nous oriente vers Robert Chabbal, directeur de la physique au CNRS qui pose immédiatement la question : «l'énergie solaire est-elle à la hauteur des besoins en énergies de l'humanité? " Notre réponse fut immédiate : «oui, et assez rapidement avec la technologie des grandes centrales solaires et des bâtiments exploitant l'énergie solaire. Pour le plus long terme, des recherches approfondies sont nécessaires en physique des solides pour les photopiles, en chimie des hautes températures pour la production d'hydrogène, en thermique et aussi en socioéconomie. »

6 Robert Chabbal décide alors, sans délais, de lancer un Programme interdisciplinaire sur l'énergie solaire (Pirdes) et propose comme projet initial la réalisation d'un prototype de grande centrale solaire thermodynamique, la centrale Themis qui fait l'objet d'un accord pour sa réalisation avec l'EDF. Peu après, il décide également de la création du Programme interdisciplinaire de recherche sur l'environnement (Piren). Les événements actuels du monde de l'énergie comme les débats autour du changement climatique attestent du rôle de pionnier qu'a joué Robert Chabbal dans cette entreprise de mise en place de projets d'envergure autour de l'énergie et de l'environnement.

7 À lire : http://histoire-cnrs.revues.org/document3062.html

Centrales solaires thermodynamiques versus photopiles

Pour dynamiser le tout premier programme interdisciplinaire du CNRS, le Pirdes, décision fut prise de l'arrimer à un grand projet: la grande centrale thermodynamique à concentration Themis. Cette décision fit l'objet de vives controverses. À l'époque, beaucoup reprochèrent au CNRS une approche qui semblait plus sortir de l'imagination de Jules Verne que tenir de la technologie contemporaine des cellules solaires. Notre réponse: il était immédiatement possible de réaliser et d'industrialiser les centrales solaires qui permettaient des usages thermiques à hautes températures, un stockage économique par changement de phase. Enfin, elles étaient susceptibles après industrialisation de délivrer un courant électrique à un prix bien plus faible que les photopiles*. Nous ajoutions qu'il était probable que, trente ans plus tard (soit donc vers 2004..., nous étions en 1974), les photopiles soient encore réalisées en coupant des tranches de 
silicium! Cette prospective s'est malheureusement avérée juste, même si aujourd'hui l'espoir de réaliser des cellules solaires à moindre coût grâce aux nanotechnologies se profile à l'horizon. En raison de l'accroissement de l'efficacité énergétique (éclairage, nouvelles technologies de l'information...) les cellules solaires demeurent une excellente solution pour des situations hors réseaux ou de microréseaux, mais les centrales solaires connaissent un intérêt renouvelé pour l'alimentation en électricité des grandes villes de la zone intertropicale bordées de déserts, dans le Maghreb, en Chine, en Australie, aux États-Unis, en Inde...

Ceux qui souhaitent en savoir plus peuvent se reporter au site de Solar Paces de l'Agence internationale de l'énergie: http://www.solarpaces.org/ où l'avenir des centrales solaires à concentration est bien détaillé. Elles pourront jouer dans le futur un rôle réel dans les bilans énergétiques.

* P. Matarasso. «Naissance des centrales solaires». Science et Avenir, spécial « énergie solaire », 1977.

8 Concentrateur parabolique du four solaire de $1000 \mathrm{~kW}$ du CNRS, à Odeillo et premier "poste de chauffage solaire » de 1,5 kW pour les très hautes températures réalisé par Félix Trombe, Marc Foëx et Charlotte Henri La Blanchetais à Meudon en 1946 avec un projecteur de DCA. (C) CNRS Photothèque/Yann Rantier

9 Véhicule solaire Solelhada, conçu et fabriqué par les équipes de l'École nationale supérieure d'électrotechnique, d'électronique, d'informatique, d'hydraulique et des télécommunications (Enseeiht). Il intègre des techniques sophistiquées tant au plan de la mécanique (moteur-roue), de l'électronique et de la puissance électrique que de l'aérodynamisme; autant d'éléments destinés à rendre ce véhicule compétitif dans l'économie d'énergie. Solelhada traverse ici l'Australie durant le World Solar Challenge 2001. (C) CNRS Photothèque/Stéphane Astier

\section{NOTES}

1. Cette histoire a été racontée dans ses aspects factuels et institutionnels dans J.

Deflandre et G. Ramunni. « Faire face à la crise du pétrole : l'énergie solaire au CNRS », in La revue pour l'histoire du CNRS, $\mathrm{n}^{\circ} 4$, mai 2001.

\section{RÉSUMÉS}

L'histoire se répète. En 2008, alors que le prix du baril flirtait allègrement avec les 150 dollars, les pays occidentaux ont dû faire face à une nouvelle crise. Déjà avec le premier choc pétrolier, ils s'étaient tournés vers des énergies de substitution. Comment la recherche sur l'énergie solaire s'est-elle développée au CNRS ? Pierre Matarasso nous livre sa version des faits. 


\section{AUTEUR}

\section{PIERRE MATARASSO}

Pierre Matarasso est directeur scientifique adjoint à l'Institut écologie et environnement (Inee) du CNRS. 